\title{
Simulation of Supply-Chain Networks: A Source of Innovation and Competitive Advantage for Small and Medium-Sized Enterprises
}

\author{
Giacomo Liotta
}

\author{
"Simulation provides cheap insurance and a cost" \\ effective decision-making tool for managers. It allows \\ us to minimize risks by letting us discover the right \\ decisions before we make the wrong ones.
}

Robert E. Shannon

Professor of Industrial Engineering

(tinyurl.com/cgqjp74)

\begin{abstract}
On a daily basis, enterprises of all sizes cope with the turbulence and volatility of market demands, cost variability, and severe pressure from globally distributed competitors. Managing uncertainty about future demand requirements and volumes in supply-chain networks has become a priority. One of the ways to deal with uncertainty is the utilization of simulation techniques and tools, which provide greater predictability of decision-making outcomes. For example, simulation has been widely applied in decision-making processes related to global logistics and production networks at the strategic, tactical, and operational levels, where it is used to predict the impact of decisions before their implementation in complex and uncertain environments. Large enterprises are inclined to use simulation tools whereas small and medium-sized enterprises seem to underestimate its advantages. The objective of this article is to emphasize the relevance of simulation for the design and management of supply-chain networks from the perspective of small and medium-sized firms.
\end{abstract}

\section{Introduction}

Simulation is a method for tackling a problem by constructing a model of the related system. It starts with an abstraction process, from which a dynamic model of the system and its logic is built. Simulation software provides the development environment in which the model is implemented, verified, validated, and experimented with to evaluate different scenarios of interest, which depend on the goals of the simulation. Researchers and companies may create their own "in house" simulation tools, although commercial software is also available and is widely used in industry. Prices vary according to feature availability, software updates, maintenance, customer service, add-on capabilities, and user friendliness.
The widespread use of simulation tools in large enterprises as well as in research institutions confirms its relevance and practical utility. However, this article emphasizes the increasing relevance of simulation in supply-chain network design and management and, more importantly, its relevance to small and mediumsized enterprises (SMEs) as a source of competitive advantage and predictive power in their decision making.

Imagine that you are the manager responsible for the logistics of a small manufacturing company that receives orders from its main customer, a large enterprise. Every single order from the large enterprise must adhere to specific quality standards and may have a different level of complexity and value. Your globally distributed (sub-)suppliers are specialized in the 


\section{Simulation of Supply-Chain Networks}

\section{Giacomo Liotta}

production of the critical sub-components you need in order to build and complete your deliveries. The procurement lead-times can strongly vary depending on market conditions, transportation, material availability, and prices. Your competitive advantage is based on the flexibility and reliability of your deliveries and on the quality of the items you provide due to your expertise in managing your suppliers, production operations, and customer-order cycle times. However, new competitors are accessing the market, and the demand for your components fluctuates due to changes in the demand of your main customer's customers. Some of your suppliers are encountering difficulties in meeting your requests due to the financial crisis. Your labour and energy costs are increasing. The value and levels of your inventory are dramatically increasing but you must be responsive in satisfying the demand.

How will you manage this complex situation in terms of supply chain configuration, capacity planning, business process improvements, and innovation needs? How will you predict the impacts of demand and leadtime uncertainty on your logistics-production performances and cash-flows throughout a reasonable time-horizon? How will you estimate the effects of introducing a new warehousing technology in your operations? Which are the interrelationships among the decision variables and their sensitivity to demand and parameter variations? How will you nourish the trust of your main customer (the large enterprise) in a way that you could maintain your reliability and competitive position within its value network? Your main customer also copes with the turbulence and volatility of market demands, cost variability, and severe pressure from globally distributed competitors. The challenge is to proactively manage the uncertainty about future demand requirements and volumes while being able to adjust or even re-engineer internal processes as well as the relationships with customers and supply-chain partners in response to sudden, unexpected changes or supply-chain disruptions.

Many firms facing these challenges use simulation in the decision-making processes related to their global logistics and production networks. It is becoming increasingly imperative for smaller firms to be able to fit the dynamic supply of their resources to the decision-making processes of their key large customers. The decision support can be performed from the strategic, tactical, or operational standpoints via the execution of "what if" analyses based on purposively designed experiments.
A number of simulation and optimization software tools for designing and managing supply-chain networks are available on the market, as illustrated in surveys by Swain (2011a; tinyurl.com/afqbbco) and Funaki (2009; tinyurl.com/axhqccw). These tools can be generalpurpose modelling environments or specific tools that are dedicated to supply-chain issues. By looking at the websites of the major vendors (as listed in the two surveys cited above), one can see that the most important customers are mainly large enterprises. SMEs can be part of extended supply-chain networks while being suppliers of both large enterprises and other SMEs, or they can autonomously compete in the market arena. However, SMEs seem to underestimate the benefits of using these software tools. One of the key messages of this article is to point out that simulation capabilities are becoming a key competitive advantage and that SMEs need to consider the potential value of simulation tools for both the efficiency of their internal operations and their ability to facilitate the decision-making processes of their key customers.

The article is organized as follows. The next section introduces the role of simulation in supply-chain networks and its usage. Thereafter, simulation techniques for logistics and production networks are presented. Next, the relevance of simulation to SMEs as an enabler for gaining competitive advantages in networked logistics-production systems and in internal processes is highlighted. The second last section addresses the potential benefits of simulation for SMEs and accessibility issues. Conclusions follow.

\section{Simulation Techniques for Logistics and Production Networks}

A supply-chain network consists of suppliers, manufacturers, distributors, and retailers linked by material and information, as well as financial flows aimed at satisfying customers' demand at the lowest cost by means of diverse business strategies, cooperation mechanisms, and network configuration models. Simulation in production and logistics can be devoted to different system levels: from the evaluation of single nodes of a network (e.g., production cell, factory, warehouse, container terminal) to an entire network of interacting nodes (e.g., a production network with the related different tiers and logistics flows). The logistics-production system under investigation is modelled and reproduced in a software environment where input, parameters, variables, and stochastic elements can mimic scenarios defined by the model developers and users. 


\section{Simulation of Supply-Chain Networks}

\section{Giacomo Liotta}

The interest in simulation usage in supply-chain management has been demonstrated in a literature review by Terzi and Cavalieri (2004; tinyurl.com/aemsojo), who noted the high number of works cited in more than 80 papers on the subject. Four basic simulation types for supply-chain management can be identified: spreadsheet simulation, system dynamics, discrete event dynamic systems simulation, and business games (Kleijnen, 2005; tinyurl.com/anzhnpp). Several simulation techniques can be used in the design and management of logistics and production processes within supplychain networks. An extended classification of simulation approaches adopted for modelling logistics and production networks simultaneously takes into account several categories, as shown in Table 1.

One of the most popular simulation techniques for modelling logistics and production networks is discreteevent simulation (DES; tinyurl.com/yzr6g2h). DES is often adopted for studying logistics, supply chains, and transportation systems (Swain, 2011b; tinyurl.com/chrq4j4), and it is particularly useful for simulating supply-chain environments (Terzi and Cavalieri, 2004; tinyurl.com/ aemsojo). Several research works include more advanced approaches that combine simulation and optimization.
Current research emphasizes the need to focus on several open issues (Liotta, 2012; tinyurl.com/d4lfuj7), including: i) the use of complex adaptive systems (tinyurl.com/4sy942) to investigate the dynamicity and selfadaptation of network structures and collaboration mechanisms in supply-chain network evolution; ii) the effectiveness and good performance of control strategies in production networks at single-plant scale using approaches inspired by biology; and iii) the consideration of bankruptcy and failures from an economics and finance viewpoint using study approaches derived from physics. However, research to date has not included aspects concerning environmental, economic, and social sustainability with comprehensive approaches - in other words including, in parallel, the simulation of transport, emissions, land use and social impacts in supply chain and production networks (Liotta, 2012).

Concerning simulation in manufacturing and business, DES is a popular technique, but it does not entail the same level of stakeholder engagement as other techniques such as system dynamics and simulation gaming (Jahangirian et al., 2010; tinyurl.com/b7znoga). DES can entail time-consuming and difficult data-gathering

Table 1. Classification of simulation approaches for logistics and production networks*

\begin{tabular}{|c|c|}
\hline Category & Description \\
\hline Simulation technique & $\begin{array}{l}\text { - } \quad \text { Discrete-event simulation (DES) } \\
\text { - } \text { System dynamics } \\
\text { - } \text { Agente Carlo simulation } \\
\text { - } \quad \text { Business simulation games } \\
\text { of different simulation techniques or as an integration of } \\
\text { optimization/mathematical modelling and simulation) }\end{array}$ \\
\hline Simulation paradigm & - Local vs. distributed simulation \\
\hline Network type & - Production network or supply-chain network \\
\hline Scale & $\begin{array}{l}\text { - Single site (single plant/facility) } \\
\text { - Multiple site (network of plants, multiple tiers, or actors } \\
\text { involved in a network) }\end{array}$ \\
\hline $\begin{array}{l}\text { Usage of interdisciplinary } \\
\text { approaches }\end{array}$ & $\begin{array}{l}\text { - Modelling approaches simultaneously involving several } \\
\text { disciplines (e.g., engineering, biology, economics, physics) }\end{array}$ \\
\hline
\end{tabular}

*Adapted from Liotta (2012; tinyurl.com/d4lfuj7) 


\section{Simulation of Supply-Chain Networks}

\section{Giacomo Liotta}

phases, whereas system dynamics makes use of standardized conceptual modelling techniques and does not need the same level of hard data as DES (Jahangirian et al., 2010). On the other hand, simulation gaming is widely applied for education and training purposes as well as for research. A promising evolutionary trend relies on the combined use of either DES and analytical models (e.g., optimization models) or discrete simulation and continuous models. This aspect can be extremely helpful to: i) optimize the number of experiments to be performed in order to achieve the desired system performance in the virtual environment; ii) embody intelligence in a simulation control logic; iii) perform comparisons between solutions statically and dynamically obtained for verification and validation purposes; and iv) include into discrete systems the effects of several phenomena described by continuous functions in the reality (Liotta, 2012; tinyurl.com/ d4lfuj7).

In terms of the simulation paradigm, distributed and parallel simulation represent a challenge because they can be suitable for simulating networked logistics and production systems with several actors/modellers involved in distributed scenarios controlled by a single company, or in scenarios with the engagement of multiple autonomous companies using different simulation tools (Liotta, 2012; tinyurl.com/d4lfuj7). Architectures such as "high-level architecture" (HLA; tinyurl.com/ b92pyfe) are fundamental enablers for this purpose. However, there is still the need to overcome some deficiencies in the technology, which hinder wide and costeffective adoption of distributed and parallel simulation in industrial practice. For simulation to be an effective decision-making aid for industrial users, cost-effective tools that produce valid models in a userfriendly manner are required. Suitable simulation technologies and architectures that enable interoperability are badly needed as well as solutions for the combined use of optimization and simulation techniques, thus facilitating the integration and interoperability of optimization and simulation engines (Liotta, 2012). Indeed, the current elaboration speed of commercial computers often does not allow rapid tests of complex optimization models in combination with simulation, although notable improvements have been made for the combination of optimization and simulation such as in the case of simulation optimization.

Simulation can help companies gain competitive advantages because it allows them to dynamically observe and rapidly predict the effects of external phenomena, stochastic events, as well as (and more importantly) system/process changes in a compressed time horizon defined by the user, without any perturbation of the existing system. For instance, by simulating the procurement of materials throughout a given time horizon in response to demand patterns that are statistically generated according to historical data, a dramatic reduction in inventory and production costs can be allowed through the prediction of the most likely sourcing needs. In time, a realistic estimate of the levels of inbound and outbound material flow can have a positive impact on cashflow, work-in-progress, stock obsolescence risks and resource usage. Again, the simulation of a new manufacturing technology or additional production resources supports the rightsizing of plants and the identification of potential resource conflicts, and it provides insights for improving the responsiveness capability to unstable market demands. Consider a further example: by simulating the vehicle routing of a freightdistribution fleet, the real capability of providing customers with on-time deliveries can be estimated together with continuous service-level improvements. In the same scenario, simulation can also allow managers to estimate the potential savings resulting from the implementation of routing optimization systems that may lead to a reduction in the distances covered and better usage of vehicle capacity. These are only a few brief examples of the simulation potential in terms of cost advantage and customer value.

Companies wishing to take advantage of the benefits of simulation must carefully choose between the various tools and techniques available. There are more than 50 simulation software tools currently available in the market (see Swain, 2011a; tinyurl.com/afqbbco); to choose between them, companies should consider the problem(s) to be tackled, data availability, software price, and effort required for data gathering as well as for system modelling. However, several additional criteria can be used with respect to the project goals. Simulation software can be evaluated on the basis of, for example: its typical applications, the markets to which the software is applied, model building features, statistical analysis tools for input distribution, output analysis tools, support/training services, user interface, and animation features. For details of these aspects, see the survey by Swain (2011a), which also includes the observation that logistics and supply chains are often included in both typical applications of software and primary markets of application. 


\title{
Simulation of Supply-Chain Networks
}

\author{
Giacomo Liotta
}

\section{Relevance of Simulation to SMEs}

SMEs are remarkable contributors to the economy worldwide. For instance, SMEs account for approximately $99 \%$ of European enterprises, $92 \%$ of which are micro-enterprises (European Commission, 2011: tinyurl.com/8hfg2bw; and 2012: tinyurl.com/2dlx5x9); the percentage of SMEs in the economy is comparable in China (Xinhua, 2012; tinyurl.com/awjnagv) and India (Government of India, 2012; tinyurl.com/c7tmfj5).

There is already some interest in the application of supply-chain network simulation tools within the context of SMEs, including interesting research insights (e.g., Albino et al., 2007: tinyurl.com/cgtzyej; Jain and Leong, 2005: tinyurl.com/blvb6tw; Byrne and Heavey, 2004: tinyurl.com/ c23y3db; Swarnkar and Harding, 2009: tinyurl.com/c9juuqf). However, the number of studies is quite limited compared to the amount of literature dedicated to supplychain network modelling scenarios for large enterprises (i.e., global companies). In many cases, these studies seem to represent research-oriented applications that may not have any practical implications from a SME manager's standpoint. Conversely, the relevance of simulation for large enterprises is evident as a tool for both designing and managing complex supply-chain networks or production systems.

The use of the various simulation techniques in SMEs can be analyzed in two different contexts: i) when SMEs are single users, for the analysis and evaluation of their internal processes, and ii) when SMEs are part of supply-chain networks that consist of other SMEs as well as large enterprises. In the first context, the use of simulation is relevant to, for example: total quality management; accelerating business process re-engineering projects (see O'Kane et al., 2007; tinyurl.com/cdad315); supplier, inventory, and demand management (thus for maintaining the competitive advantage in terms of flexibility); and rapidity and effectiveness of adaptation to changes. Simulation can be then devoted to the analysis of demand patterns and logistics/production processes with respect to key performance indicators.

In the second context, the simulation usage can be an unavoidable option due to the need for an SME to rapidly evaluate the feasibility of fulfilling orders coming from large enterprises or other partners in a supplychain network. The process performances of SMEs should be aligned to predefined levels imposed or negotiated in a large supply-chain network depending on the cooperation mechanisms or strategic alliances im- plemented (e.g., continuous replenishment, vendormanaged inventory, quick response). In these contexts, simulation represents a useful enabler for analyzing "what if" scenarios.

Existing research highlights the fact that SMEs are often unable to fully gain the advantages deriving from the implementation of supply-chain management methods and tools based on information and communication technologies (ICT) (e.g., Vaaland and Heide, 2007; tinyurl.com/c6sa5ks). The potential of ICT for supply-chain management - including analytics and advanced software tools such as simulation - is generally overlooked due to the lack of specialized skills, reduced firm size, high acquisition and implementation cost, and SME managers' confidence of the practical impact on the company performances related to their usage. However, in strongly dynamic environments characterized by a high degree of uncertainty and risk, such as networked logistics and production systems, simulation is the most suitable method for predicting system performance, resource usage, and change impact throughout time.

Unfortunately, simulation modelling and the related tool usage require specific skills and substantial effort dedicated to: problem and system definition; data collection and analysis; model implementation; verification; validation; and experiment execution. Large enterprises are quite sensitive and inclined to use simulation tools in supply-chain management. Existing literature highlights that SMEs tend to give less attention to simulation and scenario analysis tools, among other planning and control ICT tools, with respect to large enterprises (e.g., Vaaland and Heide, 2007; tinyurl.com/ c6sa5ks). Thus, a technology gap might arise, which will lead to a competitive advantage for large enterprises due to their ICT-enabled capability to plan their response to the market at lower costs and with higher service levels. It then appears that SMEs do not perceive the need to use this method as well as other techniques and software tools for supply-chain management.

O'Kane and colleagues (2007; tinyurl.com/cdad3l5) have demonstrated the relevance of simulation for SMEs through the relevant contribution of simulation to quality management and business process re-engineering focusing on production operations. Other studies (e.g., Ding et al., 2006; tinyurl.com/d6z7rjj) have discussed the potential role of SMEs in supply-chain networks while simulating business environments that may include also large enterprises . 


\section{Simulation of Supply-Chain Networks}

\section{Giacomo Liotta}

Kohn and Hüsig (2006; tinyurl.com/ap49nw6) present the case of German SMEs by discussing the modest use of computer-aided innovation tools (i.e., software technologies for supporting the creative phase of designers and process developers). In this case, the software products were previously unknown to the potential users, to whom the benefits were not clear and for whom the cost was a barrier. Virtual reality simulation was mentioned among other innovation software tools. An interesting aspect of this work is that the applicable fields for the identified innovation software categories encompass scenario management (i.e., specialized tools for generating methodical forecasts for the future) as well as holistic solutions/process engineering (i.e., solutions for facilitating the entire new product development process or, for example, dedicated portfolio/resource management). Simulation could be then included in tool categories for innovation management since it is also a support mechanism for change management; it may be an enabler for evaluating the feasibility and impacts of product and process innovations.

Although simulation can strongly contribute to the competitive advantage of large enterprises in the continuous improvement and innovation of their business processes, it seems that their potential benefits from the standpoints of SMEs are not sufficiently explored. However, they will significantly enhance their competitiveness by adding more resilience to their flexibility through the use of appropriate supporting tools for managing uncertainty. For example, in the event of a sudden, high-priority increase in order quantities and variety from a customer that will boost future sale opportunities, the prediction of the impacts of the delayed fulfillment of the orders in progress (e.g., potential penalties, material inventory on-hand), and of the additional costs for the new orders (e.g., materials to be procured, set-up costs, additional workforce needs) can help the manager of an SME to estimate and compare the costs in the new scenario. More importantly, it can allow the manager to forecast the related benefits in the medium-to-long term as well as the potential resource needs for maintaining the existing customers while fostering the company growth. Moreover, data gathering and elaboration concerning the company's internal performance can help to govern high variability levels of production and service times. In addition to the practical resource optimization supported by ICT, this manager's predictive power can be a decisive competitiveness driver. In the current, turbulent market scenarios, missing a market opportunity or failing to respect an agreed, or even expected, performance level can determine success or failure in business.
As Prahalad and Krishnan (2008; tinyurl.com/4yowma2) argue, business processes and analytics represent the mediation layers among innovation, business models, and day-to-day operations. Business processes are dynamic while analytics provide insights by elaborating data. Focused analytics can represent a unique opportunity for the identification of trends and intervention of managers. Moreover, in a ICT architecture, the two layers consisting of, respectively, proprietary and standard analytics, and ICT applications, can provide a source of competitive advantage (Prahalad and Krishnan, 2008). Therefore, since business processes and analytics can be sources of competitive advantages, simulation, as a method for modelling processes and obtaining data for complex elaborations and analyses, represents an enabler for boosting continuous improvement and innovation.

\section{The Potential Benefits of Simulation for SMEs}

An emblematic case study by Jain and Leong (2005; tinyurl.com/blvb6tw) shows how a simulation has contributed to an SME's success by contributing to its selection as supplier to a defence contractor. The readiness of the SME's simulated supply chain and of its capability to meet requirements and goals convinced the primary customers and large defence manufacturers, which then supported the contract award to the SME. Consider another case of an SME operating as supplier to a large enterprise. The SME proposed a new configuration of the procurement processes, which also involved other sub-suppliers of the large enterprise in the supply-chain network. Simulation experiments highlighted a remarkable increase in the system's stability and lower lead times while supporting the new supplychain network configuration scenario.

The accessibility of simulation modelling and related tools to SMEs is mainly affected by two factors: i) the knowledge gap related to the lack of skills and awareness of simulation advantages, and ii) the acquisition and implementation cost. The SMEs' knowledge gap related to analytics and therefore to simulation in their operations can be bridged by training and competency development. Investing in such activities can represent a fundamental step in a roadmap towards the reinforcement of a competitive advantage in the medium-tolong term. This knowledge gap can be bridged, for instance, by forms of cooperation with other SMEs that are willing to share the competency development in this field or with research institutions and educational institutions. 


\section{Simulation of Supply-Chain Networks}

\section{Giacomo Liotta}

The cost barrier can be overcome by considering new business models that can be implemented by simulation software developers, vendors and experts. As illustrated by Heikkilä and Kuivaniemi 2012; timreview.ca/article/564), among others, business ecosystems are becoming more and more knowledge-intensive, therefore the selling of systems of products and technology-enabled services will become increasingly relevant in the near future. Thus, the provision of simulation as a service could increase the accessibility of simulation tools to SMEs by reducing ownership cost (e.g., license fees) and the amount of learning, training, and skills required in the usage start-up phase. In the survey by Swain (2011a; tinyurl.com/afqbbco), the prices of simulation software ranged between $\$ 700$ USD to more than $\$ 15,000$ USD). However, the total cost of ownership throughout a certain time horizon can be relevant considering also the effort necessary for data gathering and analysis, system modelling, and experimental campaigns in the simulation environment. SMEs should evaluate this cost and strive to compare it with the cost of their traditional decision-making processes and the opportunity costs associated to the lack of these tools (e.g., in terms of time-to-market, response time to unexpected events, efficiency and effectiveness of change management processes in response to product/process innovations).

A simulation project requires both experts of the system to be studied (i.e., essential members of the organization for which the project is conducted) and experts of simulation techniques and tools. Should the organization lack the latter category, the simulation study can be developed internally by hiring a specialist or training an inside resource, or an external consultancy can be contracted (Shannon, 1998; tinyurl.com/cgqjp74). More specifically, the training of internal people may require several months and may depend on the tool selected. The company's implementation effort and time can vary widely depending on several factors such as the problem complexity, level of detail of the model, data gathering and analysis, programming needs, number of experimental campaigns, output analyses, and tool features and user-friendliness. For instance, a skilled person can implement a DES project in a matter of weeks or months, depending on the mentioned factors. Moreover, input data gathering and evaluation may require up to one third of the total time necessary for the study (Shannon, 1998). Finally, it must be underlined that economies of scale and scope can be gained if the simulation model design is oriented to the reusability, flexibility, modularity, and scalability with an efficient use of parameters and variables. Thus, relevant savings of time and effort can be obtained in further experimental campaigns and new studies.

More generally, concerning the technology-based planning and control methods in supply-chain management (including simulation and scenario analysis systems), the adoption of SMEs lacking the necessary financial, human, and other resources can be based on horizontal cooperation with other SMEs, development of vertical partnerships in the supply chain, and extension of IT providers' product ranges by including support aimed at delivering turn-key solutions for SMEs (Vaaland and Heide, 2007; tinyurl.com/c6sa5ks). On the other hand, Funaki (2009; tinyurl.com/axhqccw) discusses the business models for supply-chain design software while proposing, for example, alliances between supplychain-management consulting firms and vendors of supply-chain management software and design platforms, thereby providing an infrastructure on which companies can carry out supply-chain design and review processes as well as receive consultancy on demand.

Such business models could increase the accessibility of simulation to SMEs and the supply-chain networks intertwined in their business ecosystems would therefore benefit from improved responsiveness, reliability, and resiliency of partners. Public bodies, research institutions, associations, banking system, as well as investors can positively interact with SMEs and large enterprises in this environment that is increasingly oriented to technology-enabled business processes and innovation. The business ecosystem would be the arena where the supply-chain network becomes a real value network and where co-opetition takes place. The value co-creation based on the provision and use of simulation software tools could be then realized from two standpoints: i) users (i.e., SMEs) and ii) simulation vendors and consultants.

For users, value co-creation could be realized from opportunities to:

1. Autonomously create their own experiments and modelling customizations in the simulation environment. These opportunities occur in the context of internally performed model implementation and simulation-environment customization.

2. Exchange and co-elaborate information with supplychain network partners, for example, while testing para- 


\section{Simulation of Supply-Chain Networks}

\section{Giacomo Liotta}

meters and variables of common use, or the output and parameters of the partners' models, through, for example, the distributed-simulation paradigm. These opportunies occur in the context of internally performed model implementation and selected data sharing through interfaces and architectures for running distributed models developed by different companies.

For simulation vendors and consultants, value co-creation could be realized from opportunities to:

1. Customize the SME simulation environment on the basis of the user requirements

2. Develop new features for tools, inspired by new user requirements

\section{Operate as simulation service provider for the SME}

4. Outline and transfer change-management and innovation patterns to the simulation user on the basis of past experiences or expertise in supply-chain design or re-design projects

In this way, simulation may represent a "user toolkit for innovation" (see von Hippel 2001; tinyurl.com/3dwqxlw): in the event of simulation provided as a service, the software vendor would be, in practice, the simulation user, while the SME would be the actor exploiting the output of the simulation service. In this scenario, an SME having unclear ideas about its real innovation needs could obtain insights and focused indications for driving its innovation processes. At the same time, the simulation service provider could innovate the tools offered on the basis of new customer requirements that emerged in this interaction and are relevant to the market.

\section{Conclusion}

SMEs are remarkable contributors to the gross domestic product of both advanced and emergent economies worldwide. They may operate independently in smallscale supply chains or in larger supply-chain networks including large enterprises. Simulation can be used by SMEs at the individual level for estimating their own performance capabilities. SMEs can also make use of simulation at the supply-chain network level for comparing their own performance estimates to network-integration requirements when cooperating with large enterprises. Finally, simulation can be exploited by
SMEs for supporting their innovation-management processes. Indeed, simulation modelling relies on creativity, which is one capability for innovating.

Nonetheless, beyond the specific utility of simulation, ICT systems already represent a predisposition to the virtual test of scenario alternatives through the availability of information infrastructure and large amounts of data coming from the execution of business processes. ICT and analytics for supply-chain management and change management are enablers for the evaluation of complex decisions at strategic, tactical, and operational levels. Access to these technologies by SMEs can be hampered by knowledge and cost barriers. These hindrances may be overcome by means of cooperation and different business models involving the software industry and users in value co-creation processes within the business ecosystem. Large enterprises already benefit from the use of these tools, but SMEs should not lose this opportunity for gaining and maintaining their competitive advantages as well as for boosting their innovation capability.

\section{About the Author}

Giacomo Liotta is Assistant Professor in the Institute of Technology and Innovation at the University of Southern Denmark. His research interests concern sustainability and innovation in supply-chain networks, including the simulation of networked logistics-production systems as well as product-lifecycle management. He received a PhD in Economics and Management Engineering at the University of Rome Tor Vergata, Italy; he also holds MBA, MSc, and BSc degrees in Management and Industrial Engineering from this university. His current teaching activities focus on the design of global supply-chain networks and environmental issues related to their design and implementation. Dr. Liotta is author of more than 20 papers published in international journals, books, and conference proceedings. He has been involved in national and European co-funded research and development projects.

Citation: Liotta, G. 2012. Simulation of Supply-Chain

Networks: A Source of Innovation and Competitive

Advantage for Small and Medium-Sized Enterprises.

Technology Innovation Management Review. November 2012: 13-20. 